\title{
Pharmacogenetics and Perinatal Clinical Pharmacology: Tool or Toy?
}

\section{Karel Allegaert ${ }^{1,2 *}$}

${ }^{1}$ Department of Development and Regeneration, University Hospitals Leuven, Belgium

${ }^{2}$ KU Leuvenand Neonatal Intensive Care Unit, University Hospitals Leuven, Belgium

\begin{abstract}
The concept of personalized medicine and pharmacogenetics reflects the notion that a specific (side) effect or risk is not at random distributed in a (sub) population. This obviously holds also promises for personalized medicine in perinatal life. There are observations on the impact of pharmacogenetics on in vivo cytochrome P450 (CYP) CD6, C219 and N-Acetyl Transferase (NAT) 2 activity in early life. However, these observations are still based on genotype-phenotype concordances described in adults and - to a certain extent - still approach the infant as 'a small adult' (when does genotype-phenotype concordance appears ?).

In addition to such 'adult driven' approach, there are also potential age-specific concordances between genotype and phenotype that are only present in perinatal life: pharmacogenetic polymorphisms as predicting covariate limited to periods during development in which a genotype-phenotype concordance still exists. Such an approach holds the promise of further individualized medicine in perinatal life, but needs simultaneous availability of clinical characteristics, pharmacologic observations and polymorphisms (mother, fetus, child).
\end{abstract}

Keywords: Developmental pharmacology; Pharmacogenetics; Ontogeny

\section{Introduction}

The principles underlying the concept of personalized medicine reflect the notion that a specific (side)-effect is not at random or normally distributed throughout a (sub) population [1,2]. This obviously also holds promises for personalized medicine in perinatal life. However, integration of pharmacogenetics to improve predictions needs to take the specific characteristics of perinatal (i.e. maternal, fetal and neonatal) pharmacology into account $[1,2]$.

In essence, the fetus and early infancy are unique subpopulations. Growth and maturation are the most crucial characteristics of these subpopulations. Birth weight increases with $50 \%$ in the first 6 weeks of life, doubles in the first 4 months to be 3 times higher at the end of infancy. In this same time interval, there is a 4 -fold increase in caloric needs. Perinatal life is characterized by a very dynamic biological system with growth, maturation and extensive variability as crucial characteristics $[3,4]$.

From a clinical pharmacology perspective, the consequence of such a dynamic setting is extensive variability throughout infancy in both pharmacokinetics and pharmacodynamics with maturation ('ontogeny') and growth (weight changes) [3,4]. We hereby strongly recommend that the integration of pharmacogeneticsas another covariate to improve individual predictions in perinatal life [3,4]. However, this should not be limited to specific iso-enzyme activities with already reported polymorphisms in adults, but should also consider genotype-phenotype concordances specific to perinatal life (maternal-fetal-neonatal subpopulation).

\section{Ontogeny of Specific Iso-enzyme Activity with Known Relevant Polymorphisms in Adults}

The classification of polymorphisms related enzyme activities are usually based on the phenotypic observations collected in adults. An illustration is the cytochrome P450 (CYP) CYP2D6 activity score, translating genotype information into a qualitative measure of the phenotype in adults. Such classification results in subpopulations (e.g. poor, slow, extensive, ultrafast metabolizers) of patients that may improve individual predictability of drug-related (side) effects [5].

Genotyping of the polymorphic cytochrome P450 (CYP) 2D6 gene is used increasingly in clinical practice in adults. However, pharmacogenomics are only one group of covariates of variability. The phenotypic variability is the result of genomic variation and environment. Other clinical characteristics like co-morbidity (e.g. hepatic or renal dysfunction) or co-medication should be considered together with the genomic variation. As an illustration, the association of CYP2D6 duplication (ultrafast metabolizer) and renal impairment resulted in respiratory depression in an adult following tramadol exposure [6]. The same holds true for the interaction of ontogeny (i.e. age-dependent maturation) and polymorphisms [2-4,7].

Based on observations collected in neonates and young infants during continuous intravenous tramadol (M) administration, we were able to show that both postmenstrual age and CYP2D6 activity score were independent covariates of the variability observed. With increasing postmenstrual age, the contribution of the CYP2D6 activity score becomes more relevant (Figure 1) [8]. With increasing phenotypic activity due to ontogeny, this phenotypic activity progressively reflects pharmacogenomic related differences (Figure 1). A similar pattern of 'growing into the genotype/phenotypic correlation' has been reported for a limited number of other enzymes, including CYP2C19 and N-Acetyl Transferase (NAT) $2[9,10]$.

CYP2C19 ontogeny had a relevant impact on weight-normalized clearance of oral pantoprazole $(1.25-2.5 \mathrm{mg})$ in 33 neonates and

*Corresponding author: Karel Allegaert, Neonatal Intensive Care Unit, University Hospital, Herestraat 493000 Leuven, Belgium, Tel: 00-32-16-343850; Fax: 00-3216-343209; E-mail: karel.allegaert@uzleuven.be

Received November 14, 2012; Accepted November 25, 2012; Published November 29, 2012

Citation: Allegaert K (2012) Pharmacogenetics and Perinatal Clinica Pharmacology: Tool or Toy? J Pharmacogenom Pharmacoproteomics 3: e132. doi:10.4172/2153-0645.1000e132

Copyright: @ 2012 Allegaert K. This is an open-access article distributed under the terms of the Creative Commons Attribution License, which permits unrestricted use, distribution, and reproduction in any medium, provided the original author and source are credited. 


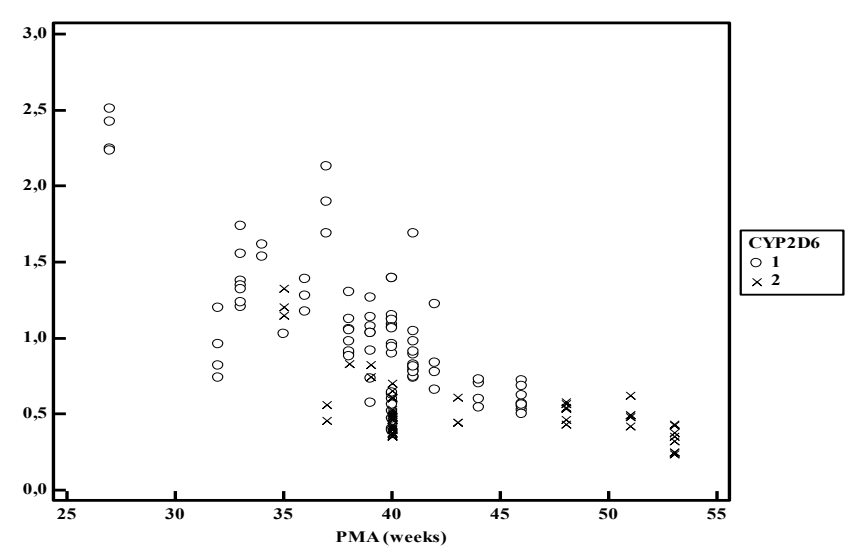

[M = tramadol, $\mathrm{M} 1=$ O-demethyl tramadol $]$

Figure 1: The impact of postmenstrual age (PMA) and CYP2D6 activity score on the plasma log M/M1 values is illustrated in neonates and young infants with an CYP2D6 activity score of either $<2$ or $\geq 2$. The lower the log M/M1 value, the higher the phenotypic CYP2D6 iso-enzyme activity. There is an PMA-dependent effect and a polymorphism dependent effect (CYP2D6 activity score of $<2$ or $\geq$ 2) [8].

infants with an age range between birth and 19 weeks of postnatal age. In essence, neonates ( $n=7, \leq 4$ weeks postnatal age) showed a phenotypic poor metabolizer CYP2C19 activity irrespective of the CYP2C19 polymorphism, with a subsequent progressive increase [9]. Two patients (one neonate, one infant of 16weeks) had a CYP2C19 genotype compatible with poor metabolizer phenotype. There was not yet a difference in pantoprazole clearance related to the CYP2C19 genotype in neonates, while the poor metabolizer infant indeed had a lower clearance compared to the other observations in infants [9].

Similarly, the role of NAT 2 ontogeny and polymorphisms on isoniazid pharmacokinetics was quantified in a cohort of infants ( $\mathrm{n}=$ 151,3-24 months postnatal age). The authors hereby described different NAT2 ontogeny profiles for each of the 3 pharmacogeneticacetylaction groups (i.e. fast acetylation $v s$ intermediate $v s$ slow) [10]. In the slow acetylation group, there was no age-related increase in phenotypic acetylation, while the difference in maturational rate between fast and intermediate acetylation groups resulted in about $30 \%$ higher clearance at 3 months ( 14.25 vs $10.88 \mathrm{~L} / \mathrm{h})$ and $45 \%$ at 24 months (22.84 vs 15.58 $\mathrm{L} / \mathrm{h}$ ) in fast acetylation cases [10].

\section{Genotype-Phenotype Concordance: A Developmental Perspective on Perinatal Pharmacology}

The 3 above discussed iso-enzyme specific examples (CYP2D6, CYP2C19, NAT 2) illustrate that the utility of pharmacogenetics as predicting covariate is limited to periods during development in which genotype-phenotype concordance already exists. However, these observations are still mainly driven based on known genotypephenotype concordance as described in adults and - to a certain extent - we still approach the infant as 'a small adult'.

Theoretically, there is the potential of age-specific concordances between genotype-phenotype in perinatal life that can only be unveil following focused evaluation in this population $[2,11,12]$. This includes the developmental context in which specific genes with known polymorphisms are active: the genotype-phenotype correlation may no longer apply beyond infancy. Fetal malformations or adverse drug reactions following maternal exposure during pregnancy or in postpartum through breastfeeding are obvious field of interest for such a genotype/phenotype exploration.

\section{Genotype-phenotype concordance: maternal-fetal pharma- cology}

Maternal consumption of acetaminophen during pregnancy has been associated with an increased risk to develop atopy in their offspring. In the Avon Longitudinal Study, nuclear erythroid 2 p45related factor 2 (Nrf2) polymorphism and glutathione S-transferase (GST, M1, T1, and P1) polymorphisms were documented in the mothers and their infants to search for genotype-phenotype concordances [13]. It was hereby documented that the antioxidant genotype of the infant did not modify associations between infant acetaminophen use and asthma phenotypes. In contrast, the increased risk of asthma and wheezing associated with late gestation acetaminophen exposure in the presence of maternal GSTM1 was further enhanced when GSTM1 was also present in the infant. Consequently, it seems that maternal antioxidant gene polymorphisms modify the relation between prenatal acetaminophen exposure and childhood asthma, strengthening evidence for a causal, polymorphisms related association.

A similar illustration, but looking for genotype/phenotype concordance following maternal acetaminophen exposure and fetal gastroshizis has been elaborated by Leeder [9]. The author hereby also stressed that besides the maternal compartment, placental transfer and metabolism, fetal drug disposition and the developmental context also contribute to the fetal concentration/time and concentration/effect profile [1].

\section{Genotype-phenotype concordance: maternal-neonatal phar- macology}

Another setting of maternal-infant life with potential pharmacogenetic polymorphisms mediated (side) effects of drugs is breast feeding. The group of Koren reported on a breastfed infant who succumbed following opioid toxicity due to exposure to morphine (the active metabolite of codeine), prescribed to his mother who was a cytochrome P450 2D6 (CYP2D6) ultrarapid metabolizer [14]. The same research group more recently reported on the links between genetic polymorphisms in mothers and their infant and the variation in response to standard doses of codeine [15]. The authors hereby explored the associations between polymorphisms in cytochrome P450 2D6 (CYP2D6), UDP-glucuronosyltransferase 2B7 (UGT2B7), P-glycoprotein (ABCB1), mu-opioid receptor (OPRM1), and catechol O-methyltransferase (COMT) genes (all involved in the codeine pathway) and central nervous depression in 111 breastfeeding mothers and their infants. A genetic model combining specific maternal risk genotypic polymorphisms (CYP2D6 and ABCB1) was associated with central nervous depression in mothers (OR 2.74; 95\% CI 1.55-4.84) and there in infants (OR 2.68; 95\% CI 1.61-4.48) [15]. The authors hereby illustrated that genetic polymorphisms can be used to improve prediction of outcome during maternal codeine therapy.

\section{Genotype-phenotype concordance: neonatal pharmacology}

This developmental context is also of relevance in neonatal life. Effective drug administration in neonates should be based on integrated knowledge concerning the evolving physiological characteristics of the treated newborn and the pharmacokinetic and pharmacodynamic characteristics of the compound administered [24]. Consequently, improved knowledge on covariates of variability - 
including pharmacogenetics - within the population are important to improve predictability of effects and side-effects.

Aminoglycoside related toxicity may hereby serve as just another example of the need for focused observations [3]. The link between toxicity and high trough aminoglycosides levels is based on historical case series, when extended interval dosing regimens for aminoglycosides were not yet implemented [16,17]. Other covariates of aminoglycoside associated toxicity are co-administration of vancomycin, nonsteroidal anti-inflammatory drugs (e.g. ibuprofen, indomethacin), peripartal asphyxia or diuretics. More recently, accumulation related aminoglycoside toxicity has been linked with the presence of megalin, a low-density lipoprotein receptor in the renal proximal tubule and in the labyrinth epithelium [16,17]. This megalin receptor also displays polymorphism [18]. Finally, several mitochondrial DNA mutations were reported to be associated with aminoglycoside-induced hearing loss. The integration of all these covariates into a predictive model to further tailor the population means risk of aminoglycoside toxicity towards an individual predictor with reasonable sensitivity and specificity is still needed. In a first attempt, Zimmerman and Lahavrecently provided a systematic analysis on the multifactorial mechanisms behind how mitochondrial DNA mutations, aminoglycosides and loud noise can potentiate ototoxicity in extremely preterm neonates [18].

\section{Pharmacogenetics Should be Tailored to Perinatal Life, not Only Mirror Findings in Adults}

The principles underlying the concept of personalized medicine and pharmacogenetics reflect the notion that a specific (side) effect or risk is not at random distributed in a population. This obviously holds promises for personalized medicine in perinatal life [1,2,7]. We have provided some observations on the impact of pharmacogenetics on in vivo cytochrome $\mathrm{P} 450$ (CYP) CD6, C219 and $\mathrm{N}$-acetyl transferase (NAT) 2 activity in early life [8-10]. However, these observations are still based on genotype-phenotype concordances described in adults and - to a certain extent - still approach the infant as 'a small adult' (when does genotype-phenotype concordance appears?).

In addition to such 'adult type' approach, there are also potential age-specific concordances between genotype and phenotype only present in perinatal life. In this way, pharmacogenetics should be tailored to clinical research questions in perinatal life, and not only mirrors findings in adults. This approach has been illustrated based on compound specific observations (acetaminophen, codeine, aminoglycosides) as reported in literature, but necessitates simultaneous availability of clinical characteristics, pharmacologic observations and polymorphisms (mother, fetus, infant).

\section{Acknowledgements}

Karel Allegaert is supported by the Fund for Scientific Research, Flanders (Fundamental Clinical Investigatorship 1800209N). There are no conflicts of interest to report.

\section{References}

1. Zhou SF (2012) Is pharmacogenomics ready for prime time? J Pharmacogenom Pharmacoproteomics 3: e126.

2. Blumenfeld YJ, Reynolds-May MF, Altman RB, El-Sayed YY (2010) Maternalfetal and neonatal pharmacogenomics: a review of current literature. J Perinatol 30: $571-579$

3. Allegaert K, Langhendries JP, van den Anker JN (2012) Educational paper: Do we need neonatal clinical pharmacologists? Eur J Pediatrdoi.

4. Smits A, Kulo A, de Hoon JN, Allegaert K (2012) Pharmacokinetics of drugs in neonates: pattern recognition beyond compound specific observations. Curr Pharm Des 18: 3119-3146.
5. Gaedigk A, Simon SD, Pearce RE, Bradford LD, Kennedy MJ, et al. (2008) The CYP2D6 activity score: translating genotype information into a qualitative measure of phenotype. Clin Pharmacol Ther 83: 234-242.

6. Stamer UM, Stüber F, Muders T, Musshoff F (2008) Respiratory depression with tramadol in a patient with renal impairment and CYP2D6 gene duplication. Anesth Analg 107: 926-929.

7. Leeder JS (2009) Developmental pharmacogenetics: a general paradigm for application to neonatal pharmacology and toxicology. Clin Pharmacol Ther 86 678-682.

8. Allegaert K, van Schaik RH, Vermeersch S, Verbesselt R, Cossey V, et al (2008) Postmenstrual age and CYP2D6 polymorphisms determine tramadol o-demethylation in critically ill neonates and infants. Pediatr Res 63: 674-679.

9. Leeder JS, Kearns GL (2012) Interpreting pharmacogenetic data in the developing neonate: the challenge of hitting a moving target. Clin Pharmaco Ther 92: 434-436.

10. Zhu R, Kiser JJ, Seifart HI, Werely CJ, Mitchell CD, et al. (2012) The pharmacogenetics of NAT2 enzyme maturation in perinatally HIV exposed infants receiving isoniazid. J Clin Pharmacol 52: 511-519.

11. Leeder JS, Mitchell AA (2007) Application of pharmacogenomics strategies to the study of drug-induced birth defects. Clin Pharmacol Ther 81: 595-599.

12. Leeder JS, Kearns GL, Spielberg SP, van den Anker J (2010) Understanding the relative roles of pharmacogenetics and ontogeny in pediatric drug development and regulatory science. J Clin Pharmacol 50: 1377-1387.

13. Shaheen SO, Newson RB, Ring SM, Rose-Zerilli MJ, Holloway JW, et al. (2010) Prenatal and infant acetaminophen exposure, antioxidant gene polymorphisms, and childhood asthma. J Allergy Clin Imunol 126: 1141-1148.

14. Koren G, Cairns J, Chitayat D, Gaedigk A, Leeder SJ (2006) Pharmacogenetics of morphine poisoning in a breastfed neonate of a codeine-prescribed mother. Lancet 368: 704

15. Sistonen J, Madadi P, Ross CJ, Yazdanpanah M, Lee JW, et al. (2012) Prediction of codeine toxicity in infants and their mothers using a nove combination of maternal genetic markers. Clin Pharmacol Ther 91: 692-699.

16. van den Anker JN, Allegaert K (2012) Pharmacokinetics of aminoglycosides in the newborn. Curr Pharm Des 18: 3114-3118.

17. Zimmerman E, Lahav A (2012) Ototoxicity in preterm infants: effects of genetics, aminoglycosides, and loud environmental noise. J Perinatol.

18. Riedemann L, Lanvers C, Deuster D, Peters U, Boos J, et al. (2008) Megalin genetic polymorphisms and individual sensitivity to the ototoxic effect of cisplatin. Pharmacogenomics J 8: 23-28. 